\title{
半干旱区沟垄集雨对玉米光合特性及产量的影响
}

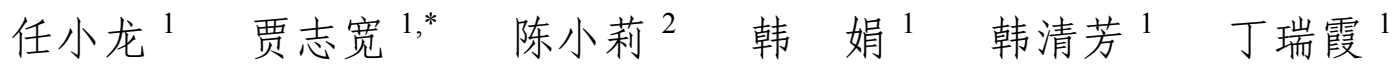 \\ $\left({ }^{1}\right.$ 西北农林科技大学干旱半干旱农业研究中心, 陕西杨凌 $712100 ;{ }^{2}$ 中国科学院水土保持研究所, 陕西杨凌 712100$)$
}

摘 要: 在中国西北黄土高原半干旱区, 沟垄集雨种植(RFPRH)系统被逐步推行, 以改善农田水分状况, 提高作物水 分利用效率 $(W U E)$, 达到高产稳产的目的。为了探索该种植方式的适宜降雨量, 在人工模拟不同降雨量下, 以传统平 作为对照, 研究了沟垄集雨种植对夏玉米土壤水分含量、功能叶片的光合参数、苂光参数和叶绿素相对含量 $(S P A D)$ 以及籽粒产量的影响。结果表明, 在 $230 \mathrm{~mm}$ 和 $340 \mathrm{~mm}$ 降雨量下, 集雨处理的净光合速率 $\left(P_{\mathrm{n}}\right)$ 、气孔导度 $\left(G_{\mathrm{s}}\right) 、$ 最大 苂光 $\left(F_{\mathrm{m}}\right) 、$ 可变菼光 $\left(F_{\mathrm{v}}\right) 、$ PS II 最大光化学量子产量 $\left(F_{\mathrm{v}} / F_{\mathrm{m}}\right) 、$ PS II 的潜在活性 $\left(F_{\mathrm{v}} / F_{\mathrm{o}}\right)$ 和光化学猝灭系数 $\left(q_{\mathrm{P}}\right)$ 均较对照 显著增加; 叶绿素相对含量(SPAD)分别提高 $17.7 \%$ 和 $13.9 \%$, 叶片瞬时水分利用效率提高 $26.9 \%$ 和 $10.1 \%$, 籽粒产量 增加 $75.4 \%$ 和 $36.7 \%$ 。在 $440 \mathrm{~mm}$ 降雨量条件下, 集雨处理的 $F_{\mathrm{m}} 、 F_{\mathrm{v}} / F_{\mathrm{m}} 、 F_{\mathrm{v}} / F_{\mathrm{o}} 、 q_{\mathrm{P}}$ 和 $S P A D$ 值在拔节期显著增加, $G_{\mathrm{s}}$ 在抽雄期显著增加, $P_{\mathrm{n}}$ 在抽雄期和孕穗期显著下降，水分利用效率在全生育期比对照平均低 $13.2 \%$, 其他各生育时期 光合生理特性及籽粒产量与对照相比差异不明显。说明沟垄集雨种植在偏旱的年份, 可显著提高玉米光合效率和叶 片水分利用效率，其全生育期适宜雨量上限可能是 $440 \mathrm{~mm}$ 。

关键词：模拟降雨；沟垄集雨种植；光合特性；夏玉米

\section{Effects of Ridge and Furrow Planting for Rainfall Harvesting on Photo- synthetic Characteristics and Yield in Corn in Semi-Arid Regions}

\author{
REN Xiao-Long ${ }^{1}$, JIA Zhi-Kuan ${ }^{1, *}$, CHEN Xiao-Li ${ }^{2}$, HAN Juan $^{1}$, HAN Qing-Fang ${ }^{1}$, and DING Rui-Xia ${ }^{1}$ \\ $\left({ }^{1}\right.$ Research Center of Agriculture in the Arid and Semi-arid Areas, Northwest A\&F University, Yangling 712100 , Shaanxi; ${ }^{2}$ Institute of Soil and Water \\ Conservation, Chinese Academy of Sciences, Yangling 712100, Shaanxi, China)
}

\begin{abstract}
Ridge and furrow planting of rainfall harvesting (RFPRH) is a cultivation technique by covering ridges with plastic films and planting crops in furrows. RFPRH has been extending in the semi-arid areas of Loess Plateau in Northwest China because it can alleviate drought stress by collecting rainfalls from the ridges and promote water use efficiency (WUE) and crop yield. To investigate the optimal quantity of rainfall in RFPRH system, we studied the effects of RFPRH, taking traditional cultivation as the control, on soil water storage, photosynthetic indexes, fluorescence parameters and chlorophyll relative content $(S P A D)$ in functional leaves, and grain yield of summer corn (Zea mays L.) under three simulated rainfall treatments. The rainfall was given 12 times (at 5-15 d interval) in corn growth duration with the total amount of 230, 340, and $440 \mathrm{~mm}$, respectively. In the RFPRH system, the net photosynthetic rate $\left(P_{\mathrm{n}}\right)$, stomatal conductance $\left(G_{\mathrm{s}}\right)$ and chlorophyll fluorescence parameters $\left(F_{\mathrm{m}}, F_{\mathrm{v}}, F_{\mathrm{v}} / F_{\mathrm{m}}, F_{\mathrm{v}} / F\right.$, and $\left.q_{\mathrm{P}}\right)$ were significantly $(P<0.05)$ higher than those in control, and the $S P A D$ value increased by $17.7 \%$ and $13.9 \%$ in 230 and $340 \mathrm{~mm}$ rainfall treatments, respectively. Compared to the control, the instantaneous WUE in leaves increased by $26.9 \%$ and $10.1 \%$, and the grain yield by $75.4 \%$ and $36.7 \%$ under 230 and $340 \mathrm{~mm}$ rainfall treatments, respectively. The $F_{\mathrm{m}}, F_{\mathrm{v}} / F_{\mathrm{m}}, F_{\mathrm{v}} / F_{\mathrm{o}}, q_{\mathrm{P}}$, and $S P A D$ value at jointing stage and $G_{\mathrm{s}}$ at heading stage increased significantly $(P<0.05)$, but $P_{\mathrm{n}}$ decreased sharply at heading and booting stages under $440 \mathrm{~mm}$ rainfall treatment in RFPRH system. The WUE of the whole growth duration of RFPRH was lower by $13.2 \%$ than that of control. The results indicate that the RFPRH system can promote $P_{\mathrm{n}}$ and leaf $W U E$ of corn in arid years, and $440 \mathrm{~mm}$ may be the upper limit of rainfall during the whole growth period in the RFPRH system.
\end{abstract}

Keywords: Rainfall simulation; Ridge and furrow planting for rainfall harvesting (RFPRH); Photosynthetic characteristics; Summer corn

基金项目：国家“十一五”科技支撑计划项目(2006BAD29B03)

作者简介: 任小龙(1981-), 男, 甘肃正宁人, 博士生, 主要从事旱地农业方面研究。Tel: 13720420365; E-mail: rxlcxl@yahoo.com.cn

“通讯作者(Corresponding author): 贾志宽。E-mail: zhikuan@tom.com

Received(收稿日期): 2007-08-08; Accepted(接受日期): 2007-12-16. 
水分是影响植物光合生理特性的一个重要因子, 干旱缺水可降低植物的光合速率 ${ }^{[1]}$ 。董合忠等 ${ }^{[2]}$ 对棉 株的研究发现, 气孔导度下降、 $\mathrm{CO}_{2}$ 反应受阻是干旱 影响光合速率的重要原因。许多研究表明, 植物体 内发出的叶绿素荧光信号包含了丰富的光合作用信 息, 可以快速、灵敏和非破坏性地分析逆境因子对 光合作用的影响 ${ }^{[3-5]}$ 。Earl 等 ${ }^{[6]}$ 认为叶绿素苂光技术 也可在大田条件下评价光合作用过程, 快速灵敏地 反映植物生理状态及其与环境的关系。

沟垄集雨种植技术 $(\mathrm{RFPRH})$ 具有显著的增产和 提高有限水分利用率的作用 ${ }^{[7-8]}$ 。通过在田间修筑沟 垄, 垄面覆膜, 沟里种植作物, 使降水由垄面向沟 内汇集, 改善作物水分供应状况, 该技术减低作物 的干旱胁迫, 能够在缺乏径流源或远离产流区的旱 平地和缓坡旱地使作物增产。Pacey 和 Cullis ${ }^{[9]}$ 指出, 在冬季降雨区, 适合于雨水集流的最低年平均降雨 量为 $100 \mathrm{~mm}$, 降雨量为 $200 \mathrm{~mm}$ 则更好, 在热带夏 季降雨区, 适合于雨水集流的最低年平均降雨量为 $150 \mathrm{~mm}$ 。

本试验针对降雨量在 $250 \sim 400 \mathrm{~mm}$ 的黄土高原 半干旱区, 利用自制模拟降雨装置, 通过模拟不同 的降雨量, 在遮雨条件下测定农田土壤水分及夏玉 米功能叶光合速率和叶绿素苂光动力学参数, 比较 不同雨量水平传统平作和沟垄种植下土壤水分状况 和夏玉米叶片光合作用的光化学过程在叶绿素水平 上的差异, 旨在为确定合理的集雨种植模式提供科 学依据。

\section{1 材料与方法}

\section{1 试验区概况及田间设计}

2006 年 6-11 月在西北农林科技大学 $\left(34^{\circ} 20^{\prime} \mathrm{N}\right.$, $\left.108^{\circ} 04^{\prime} \mathrm{E}\right)$ 农作物标本区遮雨棚内进行试验, 该地年 蒸发量 $993.2 \mathrm{~mm}$, 平均降雨量 $550 \mathrm{~mm}$, 干旱指数 $1.30 \sim 1.59$, 海拔 $466.7 \mathrm{~m}$; 年均气温 $12.9^{\circ} \mathrm{C}$, 极端高 温 $42^{\circ} \mathrm{C}$, 极端低温 $-19.4^{\circ} \mathrm{C}$, 年均日照时数 $2196 \mathrm{~h}$, 无霜期 $220 \mathrm{~d}$, 光照充足, 属暖温带半湿润偏旱型气 候。遮雨棚高 $3 \mathrm{~m}$, 呈“人”字型, 棚顶用透光塑材 覆盖, 棚侧通风(棚内光照、空气温度及湿度条件与 外界无明显差别)。供试土壤为壤土, 耕层 $(0 \sim 30 \mathrm{~cm})$ 土壤含有机质 $11.97 \mathrm{~g} \mathrm{~kg}^{-1}$ 、全氮 $1.31 \mathrm{~g} \mathrm{~kg}^{-1}$ 、全磷 $0.83 \mathrm{~g} \mathrm{~kg}^{-1}$ 、全钾 $6.18 \mathrm{~g} \mathrm{~kg}^{-1}$ 、速效氮 $53.12 \mathrm{mg} \mathrm{kg}^{-1}$ 、 速效磷 $22.34 \mathrm{mg} \mathrm{kg}^{-1}$ 、速效钾 $97.37 \mathrm{mg} \mathrm{kg}^{-1}, \mathrm{pH} 7.59$, 容重 $1.25 \mathrm{~g} \mathrm{~cm}^{-3}$ 。前茬作物为冬小麦。
田间试验采用完全区组设计，包括种植方式和 降雨量 2 个因素, 其中种植方式设 RFPRH 模式, 以 传统平作为对照; 降雨量设 230、340 和 $440 \mathrm{~mm} 3$ 个水平。小区面积为 $2 \mathrm{~m} \times 5 \mathrm{~m}$ 垄作小区 3 沟 4 垄, 沟 垄比(宽度之比)为 $1: 1$, 垄、沟宽均为 $60 \mathrm{~cm}$, 垄上 覆盖 $0.08 \mathrm{~mm}$ 厚的塑料薄膜, 产流区(垄)面积为 $2 \mathrm{~m} \times 0.6 \mathrm{~m}$, 垄高 $15 \mathrm{~cm}$ 。沟中种植玉米, 平作与 RFPRH 模式均为株距 $30 \mathrm{~cm}$ 和行距 $60 \mathrm{~cm}$, 小区单株 数为 42 株, 边行为保护行。为了防止小区水分互渗, 各 小区边缘有埋深 $2 \mathrm{~m}$ 的塑料膜, 作为水分隔离带(图 1)。

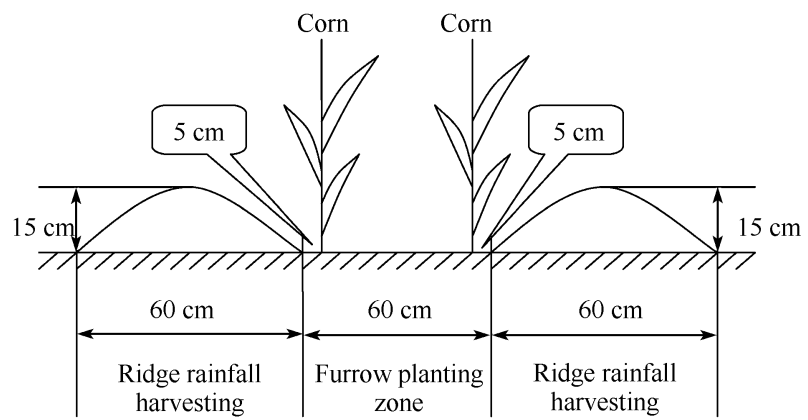

图 1 沟垄集雨种植(RFPRH)示意图

Fig. 1 Sketch map of ridge and furrow planting of rainfall harvesting (RFPRH)

供试玉米品种为户单 9 号。6 月 5 日整地、起 垄、覆膜, 基施优质农家肥 $20 \mathrm{t} \mathrm{hm}^{-2}$ 和磷酸氢二铵 $500 \mathrm{~kg} \mathrm{hm}^{-2}, 6$ 月 10 播种, 为保证出苗, 每穴先浇水 $1000 \mathrm{~mL}$, 播种 2 粒, 播深 $5 \mathrm{~cm}$ 左右。全生育期不 施追肥, 及时除草、防虫。为避免产生板结, 每次降 雨后及时松土。

\section{2 模拟降水装置及降雨量的确定}

模拟降水装置包括供水和降雨系统两部分。其 中供水系统由 1 台潜水泉、2 根外接水管(进水管和 余水排出管)以及备用储水水箱组成。降雨系统为底 部密布(每 $2 \mathrm{~cm} \times 2 \mathrm{~cm}$ 一枚) 9 号医用注射针头的铁 皮水箱(水箱上装有振动装置)及可拆卸式水箱支架, 水箱底部降雨面积为 $2 \mathrm{~m} \times 1 \mathrm{~m}$, 高度为 $0.1 \mathrm{~m}$, 外侧 安装振动装置; 支架高度为 $2 \mathrm{~m}$, 各小区两侧装置 移动轨道(高度 $0.05 \mathrm{~m}$ ), 雨滴降落高度大于 $2 \mathrm{~m}$, 雨 强为 $30 \mathrm{~mm} \mathrm{~h}^{-1}$ ，有效降雨均匀度大于 $90 \%$ 。

玉米全生育期总降雨量的确定参考中国北方半 干旱区多年降雨量及玉米生育期降雨量(图 2), 设定 为大 $(440 \mathrm{~mm}) 、$ 中 $(340 \mathrm{~mm})$ 和小 $(230 \mathrm{~mm}) 3$ 种雨量, 每次雨量范围定为 $5 \sim 50 \mathrm{~mm}$, 降雨时间间隔为 5 15 $\mathrm{d}$, 全生育期模拟降雨 12 次 (表 1)。 


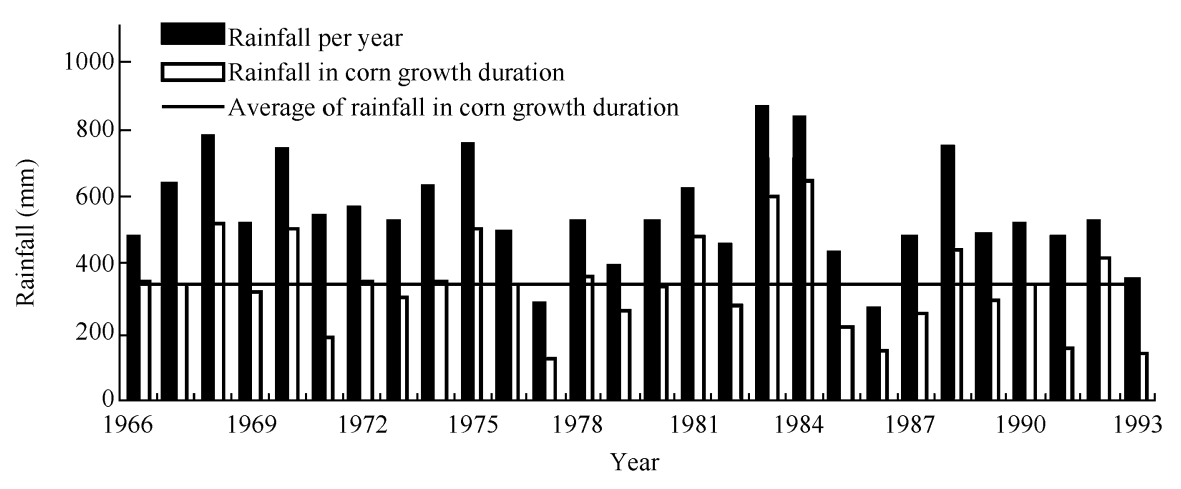

图 21966 - 1993 年杨凌区年降雨量及玉米生育期降雨量

Fig. 2 Rainfalls in whole year and corn growth duration in Yangling region from 19661993

表 1 模拟降雨的每次降雨量设置

Table 1 Quantity design of simulated rainfall

\begin{tabular}{cccccc}
\hline $\begin{array}{c}\text { 生育期 } \\
\text { Growth stage }\end{array}$ & $\begin{array}{c}\text { 降雨次数 } \\
\text { Rainfall times }\end{array}$ & $\begin{array}{c}230 \mathrm{~mm} \text { 水平 } \\
230 \mathrm{~mm} \text { level }\end{array}$ & $\begin{array}{c}340 \mathrm{~mm} \text { 水平 } \\
340 \mathrm{~mm} \text { level }\end{array}$ & $\begin{array}{c}440 \mathrm{~mm} \text { 水平 } \\
440 \mathrm{~mm} \text { level }\end{array}$ & $\begin{array}{c}\text { 降雨日期 } \\
\text { Rainfall date(month/day) }\end{array}$ \\
\hline 苗期 Seedling & 1 & 12 & 26 & 35 & $6 / 19-6 / 20$ \\
拔节期 Jointing & 3 & 25 & 35 & 50 & $6 / 28-6 / 29$ \\
& & 14 & 25 & 23 & $7 / 7-7 / 8$ \\
孕穗期 Booting & 4 & 40 & 50 & 10 & $7 / 16-7 / 17$ \\
& & 30 & 30 & 50 & $7 / 24-7 / 25$ \\
& & 20 & 12 & 50 & $8 / 8-8 / 9$ \\
成熟期 Maturity & 4 & 30 & 25 & $8 / 26$ \\
& & 25 & 40 & 40 & $9 / 3-9 / 4$ \\
& 30 & 10 & 50 & $9 / 12-9 / 13$ \\
\end{tabular}

1.3 测定项目与方法

1.3.1 光合参数 在玉米的各生长时期, 用美国 生产的 LI-6400 光合仪分别测定功能叶片(大喇叭口 期以前为第 1 片完全展开叶, 之后为棒三叶)的净光 合速率 $\left(P_{\mathrm{n}}\right)$ 、气孔导度 $\left(G_{\mathrm{s}}\right)$ 、蒸腾速率 $\left(T_{\mathrm{r}}\right)$ 和细胞间 隙 $\mathrm{CO}_{2}$ 浓度 $\left(C_{\mathrm{i}}\right)$; 测定时间为进入某一生育时期后, 第 1 次模拟降雨前 1 天, 选晴朗天气的上午 9:0011:00。叶片瞬时水分利用效率 $(W U E)$ 用 $P_{\mathrm{n}} / T_{\mathrm{r}}$ 计算 ${ }^{[10]}$ 。 1.3.2 叶绿素荧光参数 在测定光合参数的同时, 采用德国 Walz 公司制造的 Imaging-PAM 调制菼光 仪测定各生长时期玉米功能叶离体叶片的 $F_{\mathrm{o}} 、 F_{\mathrm{m}}$ 、 $F_{\mathrm{s}} 、 F_{\mathrm{m}}^{\prime}$ 和 $F_{\mathrm{o}}^{\prime}$, 并计算出苂光参数 $F_{\mathrm{v}} / F_{\mathrm{m} 、}\left(F_{\mathrm{m}}^{\prime}-F_{\mathrm{s}}\right) / F_{\mathrm{m}}^{\prime}$ 和 $q_{\mathrm{P}}$, 其中 $F_{\mathrm{v}}=F_{\mathrm{m}}-F_{\mathrm{o}} ; q_{\mathrm{P}}=\left(F_{\mathrm{m}}^{\prime}-F_{\mathrm{s}}\right) /\left(F_{\mathrm{m}}^{\prime}-F^{\prime}{ }_{\mathrm{o}}\right)$ 。每 处理 3 次重复, 测定前暗适应 $30 \mathrm{~min}$ 。

1.3.3 相对叶绿素含量 用美国产 CM-1000 非 接触式叶绿素仪测定非离体叶片相对叶绿素含量 $(S P A D)$ 。该仪器可以立即测量植物相对叶绿素含量, 其测量读数与叶片叶绿素含量具有密切相关性 ${ }^{[11]}$ 。
1.3.4 土壤水分 在 $0 \sim 120 \mathrm{~cm}$ 土层中, $0 \sim 20 \mathrm{~cm}$ 层每 $10 \mathrm{~cm}$ 取 1 个土样, $20 \mathrm{~cm}$ 以下层每 $20 \mathrm{~cm}$ 取 1 个土样, 取样位置为 RFPRH 区的沟中及平作区。采 用称重法测定, 3 次重复, 与光合参数测定同步 进行。

$W=h \times p \times b \% \times 10$ 。式中, $W$ 为土壤储水量 $(\mathrm{mm})$, $h$ 为土层深度 $(\mathrm{cm}), p$ 为土壤容重 $\left(\mathrm{g} \mathrm{cm}^{-3}\right), b$ 为土壤水 分重量百分数 $(\%)$ 。

\section{4 数据分析}

采用 DPSv3.11 软件专业版处理数据; 对测定结 果进行 $F$ 检验, 并用 $L S D$ 法进行多重比较。

\section{2 结果与分析}

\section{1 土壤水分含量}

图 3 显示, 在 3 种降雨处理中, RFPRH 区沟中 0 120 cm 土层水分平均含量均高于对照平作区, 差 异显著, 说明 RFPRH 集雨效果明显。表层土壤水分 变化比较大, $100 \mathrm{~cm}$ 土层以下, 土壤水分变化不明 
显。在拔节期, RFPRH区3 种降雨量处理的 $0 \sim 120 \mathrm{~cm}$ 土壤储水量比对照分别多 $18.3 、 13.4$ 和 $16.3 \mathrm{~mm}$, 增 加 $11.3 \% 、 9.1 \%$ 和 $10.9 \%$; 在抽雄期分别多 $14.8 、 14.2$
和 $12.9 \mathrm{~mm}$ ，增加 $7.7 \%$ 、 $8.5 \%$ 和 $8.9 \%$; 在孕穗期分 别多 $20.5 、 17.6$ 和 $12.9 \mathrm{~mm}$, 分别增加 $6.7 \% 、 7.2 \%$ 和 $6.1 \%$ 。
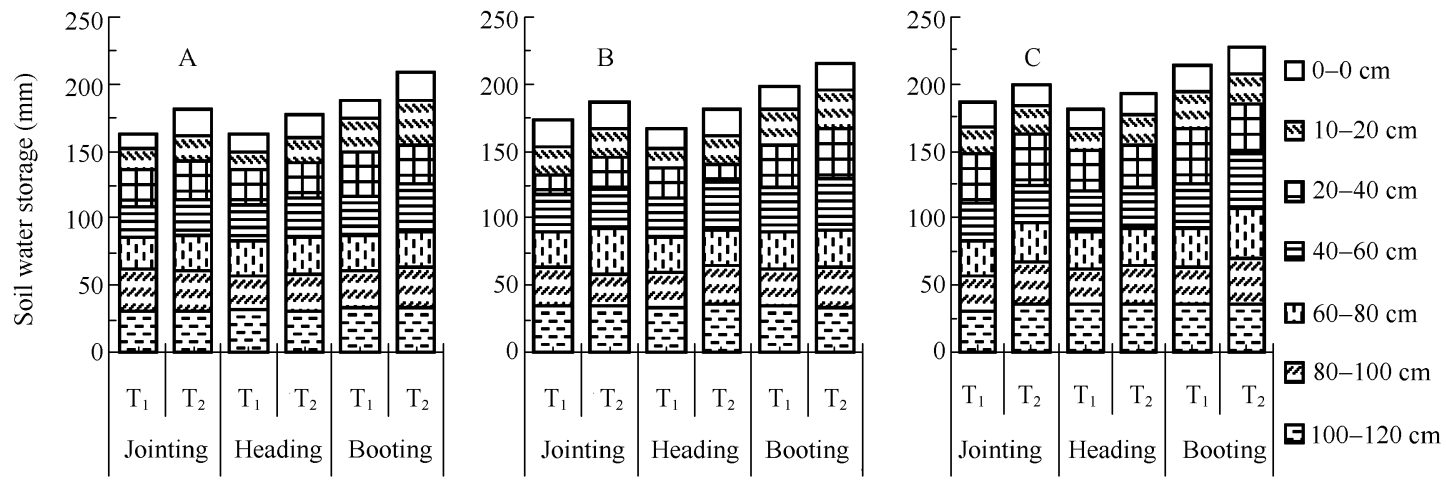

Growth stage

图 $3230(\mathrm{~A}) 、 340(\mathrm{~B})$ 和 $440 \mathrm{~mm}(\mathrm{C})$ 降雨量下 $\mathrm{RFPRH}$ 区 $\left(\mathrm{T}_{2}\right)$ 沟内和平作区 $\left(\mathrm{T}_{1}\right)$ 0 120 cm 土壤各层储水量 Fig. $30-120 \mathrm{~cm}$ soil water storing capacities in different soil layers of RFPRH $\left(T_{2}\right)$ and flat cultivation $\left(T_{1}\right)$ under 230 (A), 340 (B), and $440 \mathrm{~mm}$ (C) rainfall treatments RFPRH: ridge and furrow planting of rainfall harvesting.

2.2 不同降雨量下 RFPRH 对玉米功能叶片光合 参数的影响

2.2.1 净光合速率 $\left(P_{\mathrm{n}}\right)$ 、蒸腾速率 $\left(T_{\mathrm{r}}\right)$ 和水分利用效 率 $(W U E)$ 从表 2 可以看出, 在 $230 \mathrm{~mm}$ 降雨量 下, RFPRH 区玉米 $P_{\mathrm{n}}$ 在各生育期均显著高于平作, 而 $T_{\mathrm{r}}$ 仅在拔节期显著高于平作, $W U E$ 较平作平均 提高 $26.9 \%$; 在 $340 \mathrm{~mm}$ 降雨量下, RFPRH 区的 $P_{\mathrm{n}}$ 在各生育期也显著高于平作, 但 $T_{\mathrm{r}}$ 在两种种植模 式间没有显著差异, RFPRH 区 $W U E$ 较平作平均提
高 $10.1 \%$; 在 $440 \mathrm{~mm}$ 降雨量下, RFPRH 的 $P_{\mathrm{n}}$ 在抽 雄期和孕穗期显著低于平作, 且 $W U E$ 较平作低 $13.2 \%$ 。说明在全生育期 $230 \mathrm{~mm}$ 和 $340 \mathrm{~mm}$ 雨量下, RFPRH 模式能显著提高玉米叶片净光合速率和水 分利用效率, 尤其是 $230 \mathrm{~mm}$ 雨量下提高的幅度较 大, 而在全生育期雨量为 $440 \mathrm{~mm}$ 时, RFPRH 对夏 玉米叶片净光合速率和水分利用效率提高作用不 明显, 并在一定程度上降低了玉米叶片水分利用 效率。

表 2 不同降雨量下 RFPRH 对夏玉米净光合速率 $\left(P_{\mathrm{n}}\right)$ 、蒸腾速率 $\left(T_{\mathrm{r}}\right)$ 和水分利用效率 $(W U E)$ 的影响

Table 2 Effects of RFPRH on $P_{n}, T_{r}$, and WUE of summer corn under different rainfall treatments

\begin{tabular}{|c|c|c|c|c|c|c|c|c|c|c|}
\hline \multirow{2}{*}{$\begin{array}{l}\text { 降雨量 } \\
\text { Rainfall }\end{array}$} & \multirow{2}{*}{$\begin{array}{c}\text { 种植模式 } \\
\text { Cultivation } \\
\text { mode }\end{array}$} & \multicolumn{3}{|c|}{ 拔节期 Jointing } & \multicolumn{3}{|c|}{ 抽雄期 Heading } & \multicolumn{3}{|c|}{ 孕穗期 Booting } \\
\hline & & $P_{\mathrm{n}}$ & $T_{\mathrm{r}}$ & $\overline{W U E}$ & $P_{\mathrm{n}}$ & $T_{\mathrm{r}}$ & $W U E$ & $P_{\mathrm{n}}$ & $T_{\mathrm{r}}$ & WUE \\
\hline \multirow[t]{2}{*}{$230 \mathrm{~mm}$} & Flat & $17.60 \pm 0.73 \mathrm{~b}$ & $6.77 \pm 0.32 \mathrm{~b}$ & 2.60 & $18.77 \pm 0.82 \mathrm{~b}$ & $4.81 \pm 0.41 \mathrm{a}$ & 3.90 & $18.53 \pm 0.88 \mathrm{~b}$ & $5.09 \pm 0.39 \mathrm{a}$ & 3.64 \\
\hline & RFPRH & $24.83 \pm 0.98 \mathrm{a}$ & $7.13 \pm 0.24 \mathrm{a}$ & 3.48 & $26.13 \pm 1.02 \mathrm{a}$ & $4.97 \pm 0.22 \mathrm{a}$ & 5.26 & $21.53 \pm 0.65 \mathrm{a}$ & $5.21 \pm 0.20 \mathrm{a}$ & 4.13 \\
\hline \multirow[t]{2}{*}{$340 \mathrm{~mm}$} & Flat & $24.77 \pm 0.65 \mathrm{~b}$ & $7.05 \pm 0.17 \mathrm{a}$ & 3.51 & $18.47 \pm 0.72 \mathrm{~b}$ & $4.31 \pm 0.36 \mathrm{a}$ & 4.29 & $20.47 \pm 0.81 \mathrm{~b}$ & $4.35 \pm 0.41 \mathrm{a}$ & 4.71 \\
\hline & RFPRH & $27.37 \pm 1.22 \mathrm{a}$ & $7.14 \pm 0.23 \mathrm{a}$ & 3.83 & $22.13 \pm 0.95 \mathrm{a}$ & $4.48 \pm 0.31 \mathrm{a}$ & 4.94 & $23.67 \pm 1.01 \mathrm{a}$ & $4.73 \pm 0.29 \mathrm{a}$ & 5.00 \\
\hline \multirow[t]{2}{*}{$440 \mathrm{~mm}$} & Flat & $28.97 \pm 0.76 \mathrm{a}$ & $8.11 \pm 0.33 \mathrm{a}$ & 3.57 & $24.93 \pm 1.22 \mathrm{a}$ & $4.65 \pm 0.09 \mathrm{~b}$ & 5.36 & $22.57 \pm 0.92 \mathrm{a}$ & $5.56 \pm 0.18 \mathrm{a}$ & 4.06 \\
\hline & RFPRH & $27.93 \pm 0.65 \mathrm{a}$ & $8.32 \pm 0.12 \mathrm{a}$ & 3.36 & $22.37 \pm 0.58 \mathrm{~b}$ & $4.82 \pm 0.25 \mathrm{a}$ & 4.64 & $20.43 \pm 0.84 \mathrm{~b}$ & $6.24 \pm 0.36 \mathrm{a}$ & 3.27 \\
\hline
\end{tabular}

表中数据为 3 次重复的平均值土标准差。数据后不同字母表示两种种植方式间差异显著 $(L S D$ 法)。

Data in the table are the mean $\pm S D$ of 3 replicates. Values followed by a different letter are significantly different at $P<0.05$ according to $L S D$ test. RFPRH: ridge and furrow planting of rainfall harvesting.

\subsection{2 气孔导度 $\left(G_{\mathrm{s}}\right)$ 和胞间 $\mathrm{CO}_{2}$ 浓度 $\left(C_{\mathrm{i}}\right)$ 从表 3} 中可以看出, 在 $230 \mathrm{~mm}$ 和 $340 \mathrm{~mm}$ 雨量下, 各生育 期的 $G_{\mathrm{s}}$ 均表现为 $\mathrm{RFPRH}$ 显著高于平作, $C_{\mathrm{i}}$ 则表现为

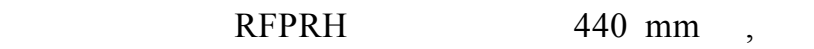

雄期和孕穗期的 $G_{\mathrm{s}}$ 为 RFPRH 显著高于平作，但全 生育期 $C_{\mathrm{i}}$ 均无显著差异。说明在全生育期 $230 \mathrm{~mm}$ 和 $340 \mathrm{~mm}$ 雨量下, RFPRH 较平作能使玉米的气孔 导度显著增加，而当全生育期雨量为 $440 \mathrm{~mm}$ 时，在 
表 3 不同降雨量下 RFPRH 对夏玉米气孔导度 $\left(G_{\mathrm{s}}\right)$ 和胞间 $\mathrm{CO}_{2}$ 浓度 $\left(C_{\mathrm{i}}\right)$ 的影响

Table 3 Effects of RFPRH on $G_{\mathrm{s}}$ and $C_{\mathrm{i}}$ of summer corn in different rainfalls treatments

\begin{tabular}{|c|c|c|c|c|c|c|c|}
\hline \multirow{2}{*}{$\begin{array}{l}\text { 降雨量 } \\
\text { Rainfall }\end{array}$} & \multirow{2}{*}{$\begin{array}{c}\text { 种植模式 } \\
\text { Cultivation } \\
\text { mode }\end{array}$} & \multicolumn{2}{|c|}{ 拔节期 Jointing } & \multicolumn{2}{|c|}{ 抽雄期 Heading } & \multicolumn{2}{|c|}{ 孕穗期 Booting } \\
\hline & & $G_{\mathrm{s}}$ & $C_{\mathrm{i}}$ & $G_{\mathrm{s}}$ & $C_{\mathrm{i}}$ & $G_{\mathrm{s}}$ & $C_{\mathrm{i}}$ \\
\hline \multirow[t]{2}{*}{$230 \mathrm{~mm}$} & Flat & $0.14 \pm 0.08 \mathrm{~b}$ & $165.77 \pm 9.34 \mathrm{a}$ & $0.13 \pm 0.02 \mathrm{~b}$ & $154.33 \pm 10.21 \mathrm{a}$ & $0.12 \pm 0.04 \mathrm{~b}$ & $146.03 \pm 12.41 \mathrm{a}$ \\
\hline & RFPRH & $0.26 \pm 0.09 \mathrm{a}$ & $142.00 \pm 11.57 \mathrm{~b}$ & $0.19 \pm 0.04 \mathrm{a}$ & $130.40 \pm 8.55 b$ & $0.18 \pm 0.03 \mathrm{a}$ & $124.50 \pm 10.35 b$ \\
\hline \multirow[t]{2}{*}{$340 \mathrm{~mm}$} & Flat & $0.18 \pm 0.05 \mathrm{~b}$ & $153.33 \pm 6.25 \mathrm{a}$ & $0.17 \pm 0.03 \mathrm{~b}$ & $164.73 \pm 9.68 \mathrm{a}$ & $0.14 \pm 0.02 \mathrm{~b}$ & $159.23 \pm 11.71 \mathrm{a}$ \\
\hline & RFPRH & $0.24 \pm 0.05 \mathrm{a}$ & $136.73 \pm 7.63 \mathrm{~b}$ & $0.24 \pm 0.04 \mathrm{a}$ & $147.27 \pm 11.30 \mathrm{~b}$ & $0.20 \pm 0.05 \mathrm{a}$ & $137.60 \pm 8.50 \mathrm{~b}$ \\
\hline \multirow[t]{2}{*}{$440 \mathrm{~mm}$} & Flat & $0.29 \pm 0.04 \mathrm{a}$ & $131.67 \pm 8.66 \mathrm{a}$ & $0.21 \pm 0.04 \mathrm{~b}$ & $155.33 \pm 8.80 \mathrm{a}$ & $0.17 \pm 0.04 \mathrm{~b}$ & $141.67 \pm 10.20 \mathrm{a}$ \\
\hline & RFPRH & $0.33 \pm 0.07 \mathrm{a}$ & $129.67 \pm 7.92 \mathrm{a}$ & $0.26 \pm 0.06 \mathrm{a}$ & $153.78 \pm 7.66 \mathrm{a}$ & $0.23 \pm 0.07 \mathrm{a}$ & $138.23 \pm 9.63 \mathrm{a}$ \\
\hline
\end{tabular}

表中数据为 3 次重复的平均值 \pm 标准差。数据后不同字母表示两种种植方式间差异显著 (LSD 法)。

Data in the table are the mean $\pm S D$ of 3 replicates. Values followed by a different letter are significantly different at $P<0.05$ according to $L S D$ test. RFPRH: ridge and furrow planting of rainfall harvesting.

增加玉米的气孔导度方面, RFPRH 较平作已没有 优势。

\section{3 不同降雨量下 RFPRH 对玉米功能叶片苂光}

\section{参数的影响}

2.3.1 初始荧光 $\left(F_{\mathrm{o}}\right)$ 、最大荧光 $\left(F_{\mathrm{m}}\right)$ 和可变荧光 $\left(F_{\mathrm{v}}\right)$ 的变化 由表 4 可知, 在 $230 \mathrm{~mm}$ 和 $340 \mathrm{~mm}$ 雨量 下，平作 $\left(\mathrm{T}_{1}\right)$ 的 $F_{\mathrm{m}}$ 在各生育时期均小于 $\operatorname{RFPRH}\left(\mathrm{T}_{2}\right)$; 在 $440 \mathrm{~mm}$ 雨量下, 平作的 $F_{\mathrm{m}}$ 除在拔节期显著低于 RFPRH 外, 其他生育时期二者差异都不显著。说明 在 $230 \mathrm{~mm}$ 和 $340 \mathrm{~mm}$ 雨量下, $\mathrm{T}_{1}$ 玉米植株 PS II 反 应中心的电子传递受到破坏, 而在 $440 \mathrm{~mm}$ 雨量下, PS II 反应中心的电子传递只有在拔节期受到一定破 坏。在 $230 \mathrm{~mm}$ 雨量下, 平作的 $F_{\mathrm{o}}$ 显著小于 $\mathrm{RFPRH}$, 说明平作植株的光化学活性明显低于 RFPRH。在 $340 \mathrm{~mm}$ 和 $440 \mathrm{~mm}$ 雨量下, $F_{\mathrm{o}}$ 在两种种植模式间没 有显著差异, 说明平作能保持较高的光化学活性, 与 RFPRH 植株光化学活性无明显差异。所以在全生 育期 $230 \mathrm{~mm}$ 和 $340 \mathrm{~mm}$ 雨量下, RFPRH 较 $\mathrm{T}_{1}$ 能改
善玉米的生长条件, 并且在 $230 \mathrm{~mm}$ 雨量下, $\mathrm{T}_{2}$ 较 $\mathrm{T}_{1}$ 的优势更强, 而当雨量增加到 $440 \mathrm{~mm}$ 时, $\mathrm{T}_{2}$ 较 $\mathrm{T}_{1}$ 无明显优势。

2.3.2 PS II 最大光化学量子产量 $\left(F_{\mathrm{v}} / F_{\mathrm{m}}\right) 、$ PS II 的潜 在活性 $\left(F_{\mathrm{v}} / F_{\mathrm{o}}\right)$ 和光化学猝灭系数 $\left(q_{\mathrm{P}}\right)$ 从表 5 可 知, 在 $230 \mathrm{~mm}$ 和 $340 \mathrm{~mm}$ 雨量下, 平作处理的 $F_{\mathrm{v}} / F_{\mathrm{m}} 、 F_{\mathrm{v}} / F_{\mathrm{o}}$ 和 $q_{\mathrm{P}}$ 在各生育时期均小于 $\mathrm{RFPRH}$ 。在 $440 \mathrm{~mm}$ 雨量下, 平作的 $F_{\mathrm{v}} / F_{\mathrm{m}} 、 F_{\mathrm{v}} / F_{\mathrm{o}}$ 和 $q_{\mathrm{P}}$ 较 RFPRH 除在玉米生长前期(拔节期)显著低于 RFPRH 外, 其 他生育时期差异都不显著。说明在 $230 \mathrm{~mm}$ 和 340 $\mathrm{mm}$ 雨量下, RFPRH 玉米植株较 $\mathrm{T}_{1}$ 具有较高的光能 转化效率, 平作最大 PS II 光能转化效率和 PS II 潜 在活性及 PS II 原初反应过程较 RFPRH 受到较为强 烈的抑制, PS II 反应中心“开放” 程度较 RFPRH 显 著减小; 在 $440 \mathrm{~mm}$ 雨量下, RFPRH 植株光能转化效 率除拔节期外在其他时期没有优势, 平作最大 PS II 光能转化效率和 PS II 潜在活性及 PS II 原初反应过 程较 RFPRH 受到的抑制弱。

表 4 不同降雨量下沟垄种植对夏玉米苂光参数的影响

Table 4 Effects of RFPRH on $F_{0}, F_{m}$, and $F_{v}$ of summer corn under different rainfall treatments

\begin{tabular}{|c|c|c|c|c|c|c|c|c|c|c|}
\hline \multirow{2}{*}{$\begin{array}{l}\text { 降雨量 } \\
\text { Rainfall }\end{array}$} & \multirow{2}{*}{$\begin{array}{c}\text { 种植模式 } \\
\text { Cultivation } \\
\text { mode }\end{array}$} & \multicolumn{3}{|c|}{ 拔节期 Jointing } & \multicolumn{3}{|c|}{ 抽雄期 Heading } & \multicolumn{3}{|c|}{ 孕穗期 Booting } \\
\hline & & $F_{\mathrm{o}}$ & $F_{\mathrm{m}}$ & $F_{\mathrm{v}}$ & $F_{\mathrm{o}}$ & $F_{\mathrm{m}}$ & $F_{\mathrm{v}}$ & $F_{\mathrm{o}}$ & $F_{\mathrm{m}}$ & $F_{\mathrm{v}}$ \\
\hline \multirow[t]{2}{*}{$230 \mathrm{~mm}$} & Flat & $0.14 \pm 0.01 \mathrm{a}$ & $0.42 \pm 0.03 \mathrm{~b}$ & $0.34 \pm 0.05 \mathrm{~b}$ & $0.16 \pm 0.02 \mathrm{a}$ & $0.38 \pm 0.06 \mathrm{~b}$ & $0.27 \pm 0.05 \mathrm{~b}$ & $0.16 \pm 0.01 \mathrm{a}$ & $0.41 \pm 0.02 \mathrm{~b}$ & $0.30 \pm 0.04 \mathrm{~b}$ \\
\hline & RFPRH & $0.12 \pm 0.02 \mathrm{~b}$ & $0.50 \pm 0.05 \mathrm{a}$ & $0.44 \pm 0.04 \mathrm{a}$ & $0.13 \pm 0.04 \mathrm{~b}$ & $0.44 \pm 0.08 \mathrm{a}$ & $0.35 \pm 0.02 \mathrm{a}$ & $0.13 \pm 0.02 \mathrm{~b}$ & $0.44 \pm 0.03 \mathrm{a}$ & $0.36 \pm 0.05 \mathrm{a}$ \\
\hline \multirow[t]{2}{*}{$340 \mathrm{~mm}$} & Flat & $0.15 \pm 0.01 \mathrm{a}$ & $0.42 \pm 0.03 \mathrm{~b}$ & $0.32 \pm 0.04 \mathrm{~b}$ & $0.14 \pm 0.03 \mathrm{a}$ & $0.49 \pm 0.06 \mathrm{~b}$ & $0.30 \pm 0.01 \mathrm{~b}$ & $0.13 \pm 0.03 \mathrm{a}$ & $0.40 \pm 0.02 \mathrm{~b}$ & $0.32 \pm 0.04 \mathrm{~b}$ \\
\hline & RFPRH & $0.16 \pm 0.03 \mathrm{a}$ & $0.44 \pm 0.01 \mathrm{a}$ & $0.36 \pm 0.03 \mathrm{a}$ & $0.12 \pm 0.03 \mathrm{a}$ & $0.53 \pm 0.02 \mathrm{a}$ & $0.36 \pm 0.04 \mathrm{a}$ & $0.11 \pm 0.02 \mathrm{a}$ & $0.45 \pm 0.04 \mathrm{a}$ & $0.38 \pm 0.06 \mathrm{a}$ \\
\hline \multirow[t]{2}{*}{$440 \mathrm{~mm}$} & Flat & $0.12 \pm 0.01 \mathrm{a}$ & $0.40 \pm 0.02 \mathrm{~b}$ & $0.36 \pm 0.03 \mathrm{~b}$ & $0.15 \pm 0.02 \mathrm{a}$ & $0.46 \pm 0.03 \mathrm{a}$ & $0.37 \pm 0.02 \mathrm{a}$ & $0.12 \pm 0.02 \mathrm{a}$ & $0.42 \pm 0.03 \mathrm{a}$ & $0.37 \pm 0.05 \mathrm{a}$ \\
\hline & RFPRH & $0.11 \pm 0.01 \mathrm{a}$ & $0.43 \pm 0.03 \mathrm{a}$ & $0.42 \pm 0.04 \mathrm{a}$ & $0.14 \pm 0.01 \mathrm{a}$ & $0.44 \pm 0.04 \mathrm{a}$ & $0.35 \pm 0.03 \mathrm{a}$ & $0.12 \pm 0.04 \mathrm{a}$ & $0.44 \pm 0.02 \mathrm{a}$ & $0.37 \pm 0.06 \mathrm{a}$ \\
\hline
\end{tabular}

表中数据为 3 次重复的平均值 \pm 标准差。数据后不同字母表示两种种植方式间差异显著 $(L S D$ 法)。

Data in the table are the mean $\pm S D$ of 3 replicates. Values followed by a different letter are significantly different at $P<0.05$ according to $L S D$ test. RFPRH: ridge and furrow planting of rainfall harvesting. 
表 5 不同降雨量下沟垄种植对夏玉米 $F_{\mathrm{v}} / \boldsymbol{F}_{\mathrm{m}} 、 \boldsymbol{F}_{\mathrm{v}} / \boldsymbol{F}_{0}$ 和 $\boldsymbol{q}_{\mathrm{P}}$ 的影响

Table 5 Effects of RFPRH on $F_{\sqrt{ }} / F_{\mathrm{m}}, F_{\mathrm{v}} / F_{\mathrm{o}}$, and $q_{\mathrm{P}}$ of summer corn under different rainfall treatments

\begin{tabular}{|c|c|c|c|c|c|c|c|c|c|c|}
\hline \multirow{2}{*}{$\begin{array}{l}\text { 降雨量 } \\
\text { Rainfall }\end{array}$} & \multirow{2}{*}{$\begin{array}{c}\text { 种植模式 } \\
\text { Cultivation } \\
\text { mode }\end{array}$} & \multicolumn{3}{|c|}{ 拔节期 Jointing } & \multicolumn{3}{|c|}{ 抽雄期 Heading } & \multicolumn{3}{|c|}{ 汓穗期 Booting } \\
\hline & & $F_{\mathrm{v}} / F_{\mathrm{m}}$ & $F_{\mathrm{v}} / F_{\mathrm{o}}$ & $q_{\mathrm{P}}$ & $F_{\mathrm{v}} / F_{\mathrm{m}}$ & $F_{\mathrm{v}} / F_{\mathrm{o}}$ & $q_{\mathrm{P}}$ & $F_{\mathrm{v}} / F_{\mathrm{m}}$ & $F_{\mathrm{v}} / F_{\mathrm{o}}$ & $q_{\mathrm{P}}$ \\
\hline \multirow[t]{2}{*}{$230 \mathrm{~mm}$} & Flat & $0.81 \pm 0.04 \mathrm{~b}$ & $2.43 \pm 0.32 \mathrm{~b}$ & $0.43 \pm 0.03 \mathrm{~b}$ & $0.71 \pm 0.06 \mathrm{~b}$ & $1.69 \pm 0.05 \mathrm{~b}$ & $0.45 \pm 0.04 \mathrm{~b}$ & $0.73 \pm 0.06 \mathrm{~b}$ & $1.88 \pm 0.05 \mathrm{~b}$ & $0.50 \pm 0.04 \mathrm{~b}$ \\
\hline & RFPRH & $0.88 \pm 0.05 \mathrm{a}$ & $3.67 \pm 0.46 \mathrm{a}$ & $0.52 \pm 0.02 \mathrm{a}$ & $0.80 \pm 0.04 \mathrm{a}$ & $2.69 \pm 0.04 \mathrm{a}$ & $0.49 \pm 0.03 \mathrm{a}$ & $0.82 \pm 0.04 \mathrm{a}$ & $2.77 \pm 0.05 \mathrm{a}$ & $0.57 \pm 0.06 \mathrm{a}$ \\
\hline \multirow[t]{2}{*}{$340 \mathrm{~mm}$} & Flat & $0.76 \pm 0.03 \mathrm{~b}$ & $2.13 \pm 0.10 \mathrm{~b}$ & $0.45 \pm 0.04 \mathrm{~b}$ & $0.61 \pm 0.06 \mathrm{~b}$ & $2.14 \pm 0.06 \mathrm{~b}$ & $0.48 \pm 0.05 \mathrm{~b}$ & $0.80 \pm 0.03 \mathrm{~b}$ & $2.46 \pm 0.06 \mathrm{~b}$ & $0.54 \pm 0.05 \mathrm{~b}$ \\
\hline & RFPRH & $0.82 \pm 0.05 \mathrm{a}$ & $2.25 \pm 0.21 \mathrm{a}$ & $0.56 \pm 0.03 \mathrm{a}$ & $0.68 \pm 0.03 \mathrm{a}$ & $3.00 \pm 0.05 \mathrm{a}$ & $0.55 \pm 0.04 \mathrm{a}$ & $0.84 \pm 0.04 \mathrm{a}$ & $3.45 \pm 0.05 \mathrm{a}$ & $0.61 \pm 0.04 \mathrm{a}$ \\
\hline \multirow[t]{2}{*}{$440 \mathrm{~mm}$} & Flat & $0.90 \pm 0.06 \mathrm{~b}$ & $3.00 \pm 0.41 \mathrm{~b}$ & $0.58 \pm 0.03 \mathrm{~b}$ & $0.80 \pm 0.04 \mathrm{a}$ & $2.47 \pm 0.04 \mathrm{a}$ & $0.63 \pm 0.05 \mathrm{a}$ & $0.82 \pm 0.03 \mathrm{a}$ & $3.08 \pm 0.04 \mathrm{a}$ & $0.63 \pm 0.04 \mathrm{a}$ \\
\hline & RFPRH & $0.98 \pm 0.04 \mathrm{a}$ & $3.82 \pm 0.34 \mathrm{a}$ & $0.64 \pm 0.04 \mathrm{a}$ & $0.80 \pm 0.06 \mathrm{a}$ & $2.50 \pm 0.03 \mathrm{a}$ & $0.62 \pm 0.04 \mathrm{a}$ & $0.84 \pm 0.05 \mathrm{a}$ & $3.08 \pm 0.06 \mathrm{a}$ & $0.65 \pm 0.05 \mathrm{a}$ \\
\hline
\end{tabular}

表中数据为 3 次重复的平均值土标准差。数据后不同字母表示两种种植方式间差异显著 ( $L S D$ 法)。

Data in the table are the mean $\pm S D$ of 3 replicates. Values followed by a different letter are significantly different at $P<0.05$ according to $L S D$ test. RFPRH: ridge and furrow planting of rainfall harvesting.

\section{4 不同降雨量下 RFPRH 对玉米功能叶 SPAD} 的影响

拔节期至孕穗期, 玉米功能叶 SPAD 随着降雨 量的递增及玉米生育期的推进而变化(图 4)。在 230 $\mathrm{mm}$ 和 $340 \mathrm{~mm}$ 雨量下, RFPRH 的 $S P A D$ 值较平作分 别增加 $17.7 \%$ 和 $13.9 \%$; 在 $440 \mathrm{~mm}$ 雨量下, RFPRH 的 $S P A D$ 值仅在拔节期显著大于平作。说明在全生 育期降雨量为 $230 \mathrm{~mm}$ 和 $340 \mathrm{~mm}$ 时, RFPRH 较平作 显著增加玉米功能叶片的叶绿素含量; 当雨量增加 到 $440 \mathrm{~mm}$ 时, RFPRH 对叶绿素含量的影响较小, 甚 至在某些生育时期没有影响。

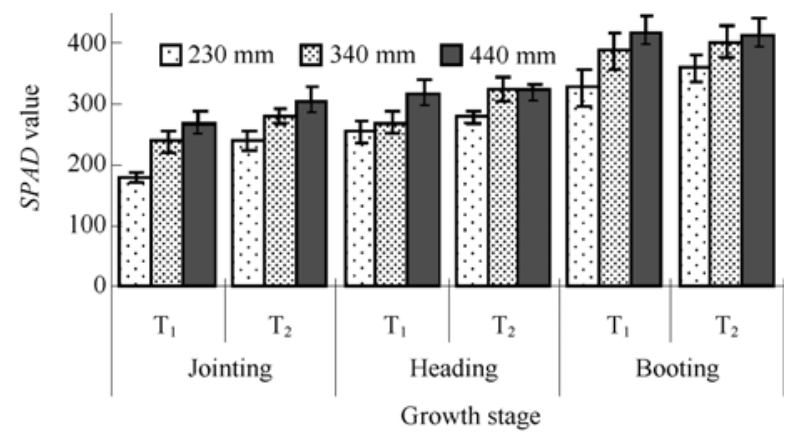

图 4 不同降雨量条件下平作 $\left(\mathbf{T}_{1}\right)$ 和 $\mathrm{RFPRH}\left(\mathrm{T}_{2}\right)$ 夏玉米功能叶的 $S P A D$ 值

Fig. 4 SPAD values of functional leaves of corn in flat cultivation $\left(T_{1}\right)$ and RFPRH $\left(T_{2}\right)$ under different rainfall treatments RFPRH: ridge and furrow planting of rainfall harvesting.

\section{5 籽粒产量}

$230 \mathrm{~mm}$ 和 $340 \mathrm{~mm}$ 雨量下, RFPRH 的籽粒产量 显著高于平作, 增幅分别为 $75.4 \%$ 和 $36.7 \%$; 当雨量 增加到 $440 \mathrm{~mm}$ 时, 两种种植方式的产量差异不显 著(图 5)。说明在夏玉米全生育期 230 440 mm 雨量 下, RFPRH 可提高玉米产量, 但其增产量随雨量增 加而趋于减小甚至消失。

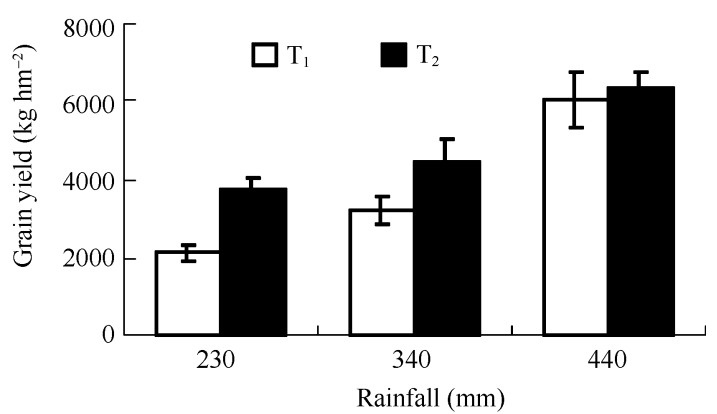

图 5 不同降雨量下 RFPRH $\left(\mathbf{T}_{2}\right)$ 与平作 $\left(\mathrm{T}_{1}\right)$ 的籽粒产量

Fig. 5 Grain yield of RFPRH $\left(T_{2}\right)$ and flat cultivation $\left(T_{1}\right)$ under different rainfall treatments

\section{3 讨论}

光合作用是一个物理、电化学及生化反应的过 程,受多种因素的影响 ${ }^{[12-13]}$, 水分胁迫是其中一个重 要因素。在水分胁迫条件下, 作物叶片光合能力降 低 ${ }^{[14-16]}$ 。殷毓芬等 ${ }^{[17]}$ 研究表明, 水分胁迫导致净光 合速率下降主要是气孔阻力增加和叶绿素含量减少 造成的, 许大全 ${ }^{[18]}$ 指出, 水分不足造成的光合速率 降低是由于水分不足引起的气孔或非气孔作用的限 制, 然而水分过多会造成土壤通气不良, 阻碍作物 根系活动，间接影响地上部光合作用。丁瑞霞等 ${ }^{[19]}$ 研究表明, 沟垄集雨种植下谷子的光合速率、蒸腾 速率、气孔导度均大于平作, 尤其是沟内边行的光 合作用强度最大。本试验当夏玉米全生育期雨量为 $230 \mathrm{~mm}$ 和 $340 \mathrm{~mm}$ 时, 与丁瑞霞等 ${ }^{[19]}$ 的结果相似。 但当雨量增加到 $440 \mathrm{~mm}$ 时, 沟垄集雨种植与平作 相比, 不但没有提高玉米叶片净光合速率和水分利 用效率, 反而在一定程度上有所降低。这可能是因 为在全生育期降水偏少的情况下，水分成了限制夏 玉米光合作用的关键因子, 沟垄作的集雨作用改善 了玉米生长的水分环境, 使其光合作用明显提高, 
并最终形成产量优势; 但当夏玉米生育期雨量较大 (440 mm) 时, 水分已经不是影响玉米光合作用的最 主要因素, 沟垄作较平作虽然明显提高了土壤水分 含量, 但不能使玉米的光合作用显著增强, 甚至可 能由于集雨作用而使种植区产生过多的水分, 影响 玉米根系的活动, 阻碍光合作用。

植物体内发出的叶绿素荧光信号极易随外界环 境而变化 ${ }^{[3,20-21]}$, 因此利用叶绿素荧光能够有效探 测许多有关植物生长发育与营养状况的信息 ${ }^{[22-23]}$, 比净光合速率 $\left(P_{\mathrm{n}}\right)$ 更能反映光合作用的真实行为。本 试验中, 叶绿素荧光参数的总体变化与净光合速率 的变化基本一致, 在玉米全生育期雨量为 $230 \mathrm{~mm}$ 和 $340 \mathrm{~mm}$ 时, 沟垄集雨种植下玉米的最大菼光 $\left(F_{\mathrm{m}}\right)$ 、可变菼光 $\left(F_{\mathrm{v}}\right) 、 \mathrm{PS}$ II 最大光化学量子产量 $\left(F_{\mathrm{v}} / F_{\mathrm{m}}\right) 、$ PS II 的潜在活性 $\left(F_{\mathrm{v}} / F_{\mathrm{o}}\right)$ 、光化学系数 $\left(q_{\mathrm{P}}\right)$ 在各生育时期较平作差异显著; 而当全生育期雨量 增加到 $440 \mathrm{~mm}$ 时, 沟垄集雨种植的玉米只有个别 苂光参数在个别生育时期与平作有差异, 整体基本 无显著差异。沟垄集雨种植技术可显著改善旱作区 作物生长的水分环境 ${ }^{[8-9]}$, 究其原因, 可能是小降雨 量下沟垄集雨种植的蓄水保墑功能缓解了玉米的水 分胁迫, 而当雨量增大为 $440 \mathrm{~mm}$ 时, 两种种植方式 下玉米的可利用水量相似, 沟垄集雨种植的水分优 势减弱或消失。

\section{4 结论}

在偏旱年份, 沟垄集雨种植可以显著提高玉米 光合效率和叶片水分利用效率, 增大叶片的叶绿素 含量, 为玉米高产提供有利的条件。当玉米生育期 雨量较大时, 沟垄集雨种植对提高玉米光合效率和 叶片水分利用效率较平作的优势减小, 甚至当雨量 增加到 $440 \mathrm{~mm}$ 时, 玉米叶片短时水分利用效率反 而降低, 所以, 沟垄集雨种植夏玉米适宜的全生育 期雨量上限可能是 $440 \mathrm{~mm}$ 。

\section{References}

[1] Qi W(綦伟), Tan H(谭浩), Zhai H(翟衡). Photosynthetic characters and fluorescence parameters of different grape stocks under water stress. Chin J Appl Ecol(应用生态学报), 2006, 17(5): 835-838(in Chinese with English abstract)

[2] Dong H-Z(董合忠), Li W-J(李维江), Tang W(唐薇), Li Z-H(李振怀), Zhang D-M(张冬梅). Effects of water-deficit and water-logging on some physiological characteristics of cotton seedlings, Acta Bot Boreal-Occident Sin(西北植物学 报), 2003, 23(10): 1695-1699(in Chinese with English ab- stract)

[3] Krause G H, Weis E. Chlorophyll fluorescence and photosynt hesis. Annu Rev Plant Physol Plant Mol, 1991, 42: 313-349

[4] Barry A L. Chlorophyll fluorescence: a signature of photosynthesis. Torrey Bot Soc, 2005, 132: 650

[5] Kevin O. Imaging of chlorophyll a fluorescence: theoretical and practical aspect $\mathrm{s}$ of an emerging technique for the monitoring of photosynthetic performance. $J$ Exp Bot, 2004, 55: 1195-1205

[6] Earl H J, Tollenaar M. Using chlorophyll fluorometry to compare photosynthetic performance of commercialmaize (Zea mays L.) hybrids in the field. Field Crops Res, 1999, 61: 201-210

[7] Li X Y, Gong J D, Gao Q Z, Li F R. Incorporation of ridge and furrow method of rainfall harvesting with mulching for crop production under semiarid conditions. Agric Water Manag, 2001, 50: 173-183

[8] Li X Y, Gong J D. Effects of different ridge/furrow rations and supplemental irrigation on crop production in ridge and furrow rainfall harvesting system with mulches. Agric Water Manag, 2002, 54: 243-254

[9] Pacey A, Cullis A. Rainwater Harvesting: the Collection of Rainfall and Runoff in Rural Areas. London: WBC Print Ltd., 1989

[10] Bierhuizen J F, Slatyer R O. Effects of atmospheric concentration of water and $\mathrm{CO}_{2}$ in determining transpiration photosynthesis relationships of cotton leaves. Agric Meteorol, 1965, 2: $229-270$

[11] Peng S, Garcia F V, Laza R C. Adjustments for specific leaf weight improve chlorophyll meter's estimate of rice leaf nitrogen concentration. Agron J, 1993, 85: 987-990

[12] Zheng G-S(郑国生), Zou Q(邹琦). A study on the diurnal variation of photosynthesis of field-grown soybean in different weather conditions. Sci Agric Sin(中国农业科学),1993, 26(1): 45-50 (in Chinese with English abstract)

[13] Lin J-K(林金科), Lai Z-M (赖志明). Preliminary analysis for the relationship between eco-physiological factors and net photosynthetic rate of tea leaves. Acta Agron Sin(作物学报), 2000, 26(1): 45-50(in Chinese with English abstract)

[14] Chen J(陈军), Dai J-Y(戴俊英). Effect of drought on photosynthesis and grain yield of corn hybrids with different drought tolerance. Acta Agron Sin(作物学报), 1996, 22(6): 757-762 (in Chinese with English abstract)

[15] Wu Y-Y(武玉叶), Li D-Q(李德全), Zhao S-J(赵世杰), Zou $\mathrm{Q}$ (邹琦). Osmotic adjustment and photosynthesis of wheat leaves under soil water stress. Acta Agron Sin (作物学报), 1999, 25(6): 752-758 (in Chinese with English abstract)

[16] Hirasawa T, Hsiao T C. Some characteristics of reduced leaf 
photosynthesis at midday in maize growing in the field. Field Crops Res, 1999, 62: 53-62

[17] Yin Y-F(殷毓芬), Zhang C-L(张存良), Yao F-X(姚凤霞). Relationship of leaf photosynthetic rate with stomatal resistance and stomatal conductance to $\mathrm{CO}_{2}$ in wheat cultivars. Acta Agron Sin(作物学报), 1995, 21(5): 561-567 (in Chinese with English abstract)

[18] Xu D-Q(许大全). Photosynthesis Efficiency(光合作用效率). Shanghai: Shanghai Scientific and Technical Publisher, 2002(in Chinese)

[19] Ding R-X(丁瑞霞), Jia Z-K(贾志宽), Han Q-F(韩清芳), Ren G-X(任广崟金), Wang J-P(王俊鹏). Border effect and physiological characteristic responses of foxtail millet to different micro-catchment stripshapes in semiarid region of south Ningxia. Sci Agric Sin(中国农业科学), 2006, 39(3): 494-501 (in Chinese with English abstract)

[20] Van Kooten O, Snel J F H. The use of chlorophyll nomenclature in plant stress physiology. Photosynthesis Res, 1990, 25: $147-150$

[21] Herppich W B, Peckmann K. Responses of gas exchange, photosynthesis, nocturnal acid accumulation and water relations of aptenia cordifolia to short-term drought and rewatering. J Plant Physiol, 1997, 150: 467-474

[22] Maxwell K, Johnson G N. Chlorophyll fluorescence: a practical guide. $J$ Exp Bot, 2000, 51: 659-668

[23] Zhang W-F(张旺锋), Gou L(勾玲), Wang Z-L(王振林), Li S-K(李少昆), Yu S-L(余松烈), Cao L-P(曹连莆). Effect of nitrogen on chlorophyll fluorescence of leaves of highyielding cotton in Xinjiang. Sci Agric Sin(中国农业科学), 2003, 36(8): 893-898 (in Chinese with English abstract) 